\title{
Optimal Incomplete-Block Designs with Low Replication: A Unified Approach Using Graphs
}

\author{
R. A. Bailey ${ }^{1}$ (D) Alia Sajjad ${ }^{2}$
}

Accepted: 8 September 2021 / Published online: 8 November 2021

(c) The Author(s) 2021

\begin{abstract}
An incomplete-block design defines both a concurrence graph and a Levi graph. Properties of either graph can be used to compare designs with respect to D-optimality and with respect to A-optimality. In this paper, we show that optimality of the design implies strong conditions on connectivity properties of the graph, and use this to classify the optimal designs when the number of observational units is close to minimal.
\end{abstract}

Keywords Block design · A-optimality · D-optimality · Concurrence graph · Levi graph

Mathematics Subject Classification $62 \mathrm{~K} 05 \cdot 62 \mathrm{~K} 10 \cdot 05 \mathrm{~B} 05 \cdot 05 \mathrm{C} 40$

\section{Introduction}

The purpose of this paper is to give a unified approach to the use of graph theory to find optimal incomplete-block designs with very low average replication. Some of the results given have already been proved by graph-theoretical or other methods.

The original online version of this article was revised: We regret the stylistic deficiencies in the earlier version. The current version represents the final version.

This article is part of the topical collection "Special Issue: State of the art in research on design and analysis of experiments" guest edited by John Stufken, Abhyuday Mandal, and Rakhi Singh.

$\triangle$ R. A. Bailey

rab24@st-andrews.ac.uk

Alia Sajjad

alia.sajjad@mcgill.ca

1 School of Mathematics and Statistics, University of St Andrews, St Andrews, Fife KY16 9SS, UK

2 Department of Mathematics and Statistics, McGill University, 805 Sherbrooke St W, Montreal, QC H3A 0B9, Canada 
Therefore a part of this paper gives historical notes on how graph theory has been used to determine optimal designs, while the other part continues this approach to give new results.

The two graphs associated with an incomplete-block design are its Levi graph and its concurrence graph. In [8], Bapat and Dey used the Levi graph to find D-optimal designs with the minimal number of observations, while Balasubramanian and Dey used it in [7] to find D-optimal designs with nearly minimal number of observations. In [33], Tjur used it to find A-optimal designs with nearly minimal number of observations when the block size is two, and Krafft and Schaefer extended this in [22] to arbitrary block size.

On the other hand, Cheng formulated D-optimality in terms of the concurrence graph in [11]. In [1], Bailey used this graph to find A-optimal and D-optimal designs when the block size is two and the average replication is low. Sajjad used it in [31] to find A- and D-optimal designs when the number of observations is minimal or nearly minimal.

Section 2 of this paper recalls definitions of block designs and related concepts, including the information matrix, the Laplacian matrix, D-optimality and A-optimality, and gives the notation used for the rest of the paper. The Levi graph and concurrence graph are defined, and examples are given.

Section 3 shows how the values of both optimality criteria can be determined from properties of either the concurrence graph or the Levi graph. Then Sect. 4 uses this to give some general results for D-optimal designs and A-optimal designs. Although the results are different for the two sorts of optimality, they both show that graphs associated with optimal designs have strong constraints on whether they can be disconnected by the removal of a single edge or a single vertex. Then Sects. 5 and 6 apply these results in a unified way to classify the optimal designs when the number of observational units is very low.

\section{Definitions and Notation}

\subsection{Incomplete-Block Designs and Associated Matrices}

Consider $v$ treatments to be tested in $b$ blocks of size $k$, where $2 \leq k<v$. Such a design is an incomplete-block design. A block design is said to be connected if all contrasts among treatment effects can be estimated, under the assumption that the expected value of each observation is the sum of an unknown fixed treatment effect and an unknown fixed block effect. The number of observations is $b k$, so a necessary condition for a block design to be connected is $b k \geq b+v-1$. Put $c=b k-b-v+1$. We need $c \geq 0$. Therefore $b(k-1) \geq v-1$, and so $b \geq(v-1) /(k-1)>1$ because $k<v$.

Thus the number of observations is said to be minimal if $c=0$ and nearly minimal if $c=1$. The class of connected designs for given values of $v, b, k$ and $c$ is denoted $\mathscr{D}_{c}(v, b, k)$ in $[7,13]$. The optimal designs with minimal or nearly minimal number of observations can be of special interest to a statistician. Two block designs in $\mathscr{D}_{1}(18,6,4)$ are shown in Fig. 1. 


\begin{tabular}{|c|c|c|c|c|c|c|c|c|c|c|c|}
\hline 1 & 2 & 3 & 4 & 5 & 6 \\
2 & 3 & 4 & 5 & 6 & 1 \\
7 & 9 & 11 & 13 & 15 & 17 \\
8 & 10 & 12 & 14 & 16 & 18 \\
\hline
\end{tabular}

(a)

Fig. 1 Two block designs with $b=6, k=4$ and $v=18$

We denote individual treatments by small Roman letters such as $i$ and $j$, and individual blocks by large Greek letters such as $\Gamma$ and $\Delta$. Denote by $n_{i} \Gamma$ the number of times that treatment $i$ occurs in block $\Gamma$. The design is said to be binary if $n_{i} \leq 1$ for all $i$ and $\Gamma$. Denote by $r_{i}$ the number of observations on treatment $i$ : thus

$$
r_{i}=\sum_{\Gamma} n_{i \Gamma} .
$$

Definition 1 The incidence matrix $\mathbf{N}$ of an incomplete-block design is the $v \times b$ matrix whose $(i, \Gamma)$ entry is $n_{i} \Gamma$.

Definition 2 Given an incomplete-block design, the concurrence $\lambda_{i j}$ of treatments $i$ and $j$ is defined by

$$
\lambda_{i j}=\sum_{\Gamma} n_{i \Gamma} n_{j \Gamma} .
$$

If the design is binary, then $\lambda_{i i}=r_{i}$ and, for $i \neq j$, the concurrence $\lambda_{i j}$ is the number of blocks in which $i$ and $j$ both occur. The concurrence matrix $\boldsymbol{\Lambda}$ is the $v \times v$ matrix with $(i, j)$ entry equal to $\lambda_{i j}$. Therefore $\boldsymbol{\Lambda}=\mathbf{N N}^{\top}$.

Let $\mathbf{R}$ be the $v \times v$ diagonal matrix with diagonal entries $r_{1}, \ldots, r_{v}$, and denote by $\mathbf{I}_{b}$ the identity matrix of order $b$. By standard linear model theory, the Fisher information matrix for estimating both treatment and block parameters is $\tilde{\mathbf{C}}$, where

$$
\tilde{\mathbf{C}}=\left[\begin{array}{cc}
\mathbf{R} & \mathbf{N} \\
\mathbf{N}^{\top} & k \mathbf{I}_{b}
\end{array}\right] .
$$

The Fisher information matrix $\mathbf{C}$ for the estimation of treatment effects only is given by $\mathbf{C}=\mathbf{R}-k^{-1} \boldsymbol{\Lambda}$. For all the treatment contrasts to be estimable, the rank of $\mathbf{C}$ has to be $v-1$. See $[3,4,29,32,33]$.

\subsection{Graphs Defined by Block Designs}

We will discuss two graphs associated with block designs, the Levi graph and the concurrence graph. The Levi graph was introduced by Levi [24] and implicitly used by Bose in [9] in coining the concept of connectivity of a block design. In [12], Coxeter named it the Levi graph. Some authors call it the incidence graph. The concurrence graph was defined in [28] by Patterson and Williams as the variety-concurrence graph. It is worth mentioning here that Coxeter had called it the Menger graph in [12], and 
that Grünbaum claimed in [17] that 'The earlier "Menger graphs" ... are much less useful.'

Definition 3 The Levi graph associated with an incomplete-block design is a bipartite graph with $v+b$ vertices. The vertices in one part are labelled by the treatments, those in the other part by the blocks. There are $b k$ edges, one corresponding to each observation. An observation on treatment $i$ in block $\Gamma$ gives an edge between vertices $i$ and $\Gamma$. Thus there are $n_{i}$ edges between $i$ and $\Gamma$, and $b k$ edges in total. If the design is binary, then there is no more than one edge between any two vertices.

In any graph, the degree of a vertex denotes the number of edges incident with it. Thus in the Levi graph the degree $\operatorname{deg}(\Gamma)$ of each block-vertex $\Gamma$ is equal to $k$, while for each treatment-vertex $\operatorname{deg}(i)=r_{i}$. The design can be recovered from its incidence matrix $\mathbf{N}$ if its rows and columns are labelled and equivalently from its Levi graph if the vertices are labelled.

In a general graph with $n$ vertices and $e$ edges, the cycle rank is defined to be $e-n+1$. This must be non-negative if the graph is connected. The Levi graph has $b+v$ vertices and $b k$ edges, so $c$ is equal to the cycle rank of the Levi graph. Henceforth, we shall call it the cycle rank of the block design.

Figures 2 and 3 show the Levi graphs of the block designs in Fig. 1a, b, respectively. Throughout this paper we use the convention that an open square represents a blockvertex and a filled circle represents a treatment-vertex.

Definition 4 The concurrence graph of an incomplete-block design is a graph whose vertices are labelled by the treatments. Distinct treatments $i$ and $j$ are joined by $\lambda_{i j}$ edges.

In the concurrence graph, the degree of vertex $i$ is given by $\operatorname{deg}(i)=\sum_{\{j \mid j \neq i\}} \lambda_{i j}$. In general, two different block designs may have the same concurrence graphs: therefore

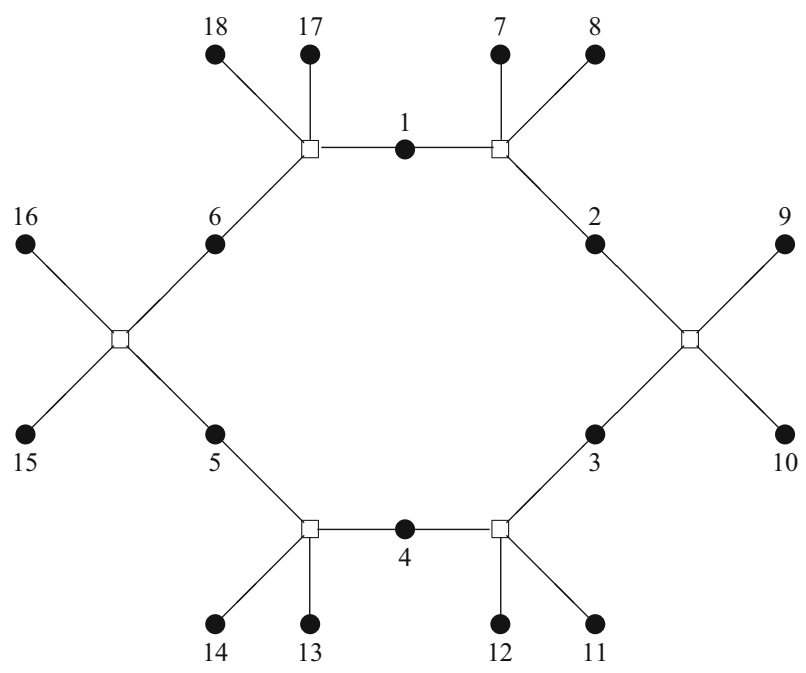

Fig. 2 Levi graph for the block design in Fig. 1a 


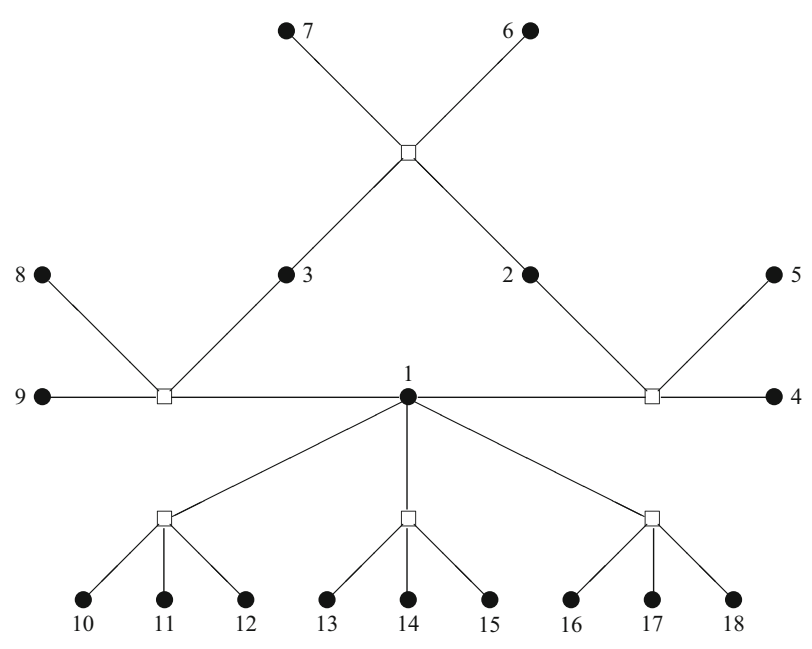

Fig. 3 Levi graph for the block design in Fig. 1b

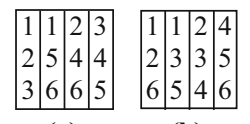

(a)

(b)

Fig. 4 Two block designs with $b=4, k=3$ and $v=6$

the block design cannot be retrieved from its concurrence graph. For example, Fig. 4 shows two block designs with $v=6, b=4$ and $k=3$ whose concurrence graph is the one shown in Fig. 5. In spite of this, the concurrence graph provides significant information to rank designs on different optimality criteria, as discussed by Bailey and Cameron in $[3,4]$. Furthermore, when $k=2$, binary block designs are effectively the same as their concurrence graphs, with each edge corresponding to a block.

The following are equivalent: (i) an incomplete-block design is connected; (ii) its Levi graph is a connected graph; (iii) its concurrence graph is a connected graph. See $[3,4,33]$.

Fig. 5 Concurrence graph for both designs in Fig. 4

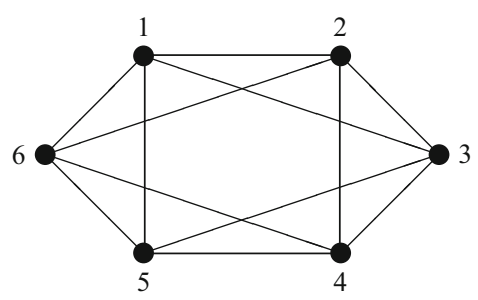




\subsection{Laplacian Matrices of Graphs}

Definition 5 Let $H$ be a graph on $n$ vertices. The Laplacian matrix $\mathbf{L}(H)$ of this graph is the $n \times n$ matrix whose $(i, j)$ entry $L_{i j}$ is the negative of the number of edges between vertices $i$ and $j$, if $i \neq j$, and whose $(i, i)$ entry $L_{i i}$ is the degree of $i$.

It follows from the above definition that $L_{i i}=-\sum_{\{j: j \neq i\}} L_{i j}$. Therefore the row and column sums of $\mathbf{L}(H)$ are all zero. Hence the Laplacian matrix $\mathbf{L}(H)$ has eigenvalue zero on the all-1 vector. Moreover, the multiplicity of this eigenvalue is equal to the number of connected components of $H$ : see [3]. The other eigenvalues of $\mathbf{L}(H)$ are all positive. One way of proving this is to note that $\mathbf{L}(H)$ is the sum of matrices $\mathbf{L}_{e}(H)$ over all edges $e$ of $H$, where the submatrix of $\mathbf{L}_{e}(H)$ corresponding to the two vertices joined by $e$ is

$$
\left[\begin{array}{rr}
1 & -1 \\
-1 & 1
\end{array}\right]
$$

and all other entries of $\mathbf{L}_{e}(H)$ are zero: see [3]. It follows that $\mathbf{L}(H)$ is positive semidefinite.

Let $G$ be the concurrence graph of a given incomplete-block design, and let $\tilde{G}$ be its Levi graph. Let $\mathbf{L}$ and $\tilde{\mathbf{L}}$ be the corresponding Laplacian matrices $\mathbf{L}(G)$ and $\mathbf{L}(\tilde{G})$. In [14-16], Gaffke showed that $\mathbf{L}=k \mathbf{C}$ and

$$
\tilde{\mathbf{L}}=\left[\begin{array}{cc}
\mathbf{R} & -\mathbf{N} \\
-\mathbf{N}^{T} & k \mathbf{I}_{b}
\end{array}\right]
$$

It was shown in $[4,33]$ that if the block parameters in the usual linear model are replaced by their negatives, then $\tilde{\mathbf{C}}=\tilde{\mathbf{L}}$.

We are interested only in connected incomplete-block designs whose blocks all have the same size. For such designs, zero has multiplicity one as an eigenvalue of $\mathbf{C}$ and $\mathbf{L}$. The other eigenvalues of these matrices will be called non-trivial.

\subsection{Optimality in Incomplete-Block Designs}

These definitions are taken from [32].

Definition 6 An incomplete-block design for $v$ treatments in $b$ blocks of size $k$ is called $D$-optimal if it maximizes the geometric mean of the non-trivial eigenvalues of the information matrix $\mathbf{C}$ over all connected block designs for $v$ treatments with $b$ blocks of size $k$.

Equivalently, a D-optimal design minimizes the volume of the ellipsoid of confidence for the vector of treatment parameters. Equivalently again, a D-optimal design maximizes the geometric mean of the non-trivial eigenvalues of the Laplacian matrix $\mathbf{L}$. 
Definition 7 An incomplete-block design is called A-optimal if it maximizes the harmonic mean of the non-trivial eigenvalues of the information matrix $\mathbf{C}$ over all connected block designs for $v$ treatments with $b$ blocks of size $k$.

Equivalently, an A-optimal design minimizes the sum of the variances of the estimates of the differences between two treatment parameters. Equivalently again, an A-optimal design maximizes the harmonic mean of the non-trivial eigenvalues of the Laplacian matrix $\mathbf{L}$.

For both D- and A-optimality, the first equivalence is given in $[29,32]$ and the second follows from the fact that $\mathbf{L}=k \mathbf{C}$.

\section{Graphs and Optimality}

\subsection{Spanning Trees}

Definition 8 A subset of the edges in a graph is a cycle if, together with the vertices at their ends, they form a connected subgraph in which every vertex has degree two. A subset of the edges (together with its vertices) is a tree if it is connected but contains no cycles. A tree whose vertices are all those of the original graph is a spanning tree.

Thus a connected graph is a tree if and only if $c=0$, while, if $c=1$, then it contains a single cycle.

Figure 6 shows a graph which is a tree: it has six vertices, five edges, and cycle rank zero. On the other hand, the Levi graph in Fig. 2 has a single cycle, which has length 12 . Every spanning tree of this graph is made by removing a single edge of the cycle from the graph.

If a graph is not connected, it has no spanning trees. In [14], Gaffke showed the relevance to optimal design theory of the following theorem, known as the matrix tree theorem, which was given by Kirchhoff [21].

Theorem 1 If $H$ is a connected graph with $n$ vertices, then the product of $n$ and the number of spanning trees of $H$ is equal to the product of the non-trivial eigenvalues of $\mathbf{L}(H)$.

In [30], Sachs gave the next theorem, which was cited in [16].

Theorem 2 Let $G$ and $\tilde{G}$ be the concurrence graph and Levi graph, respectively, of an incomplete-block design for $v$ treatments in b blocks of size $k$. Then the number of spanning trees of $\tilde{G}$ is equal to the product of $k^{b-v+1}$ and the number of spanning trees of $G$.

Fig. 6 A tree with six vertices and five edges

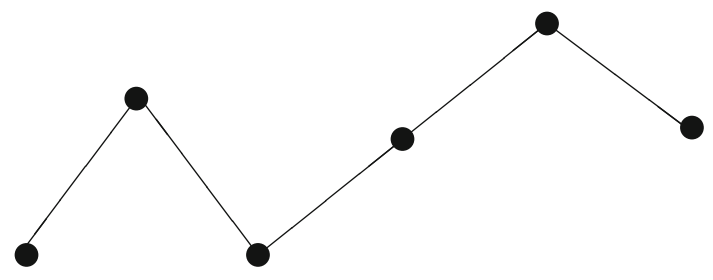


Since the product of the non-trivial eigenvalues of $\mathbf{L}$ is their geometric mean raised to the power $v-1$, these results give the following way of assessing D-optimality by using graphs.

Theorem 3 Consider a connected incomplete-block design d for $v$ treatments in $b$ blocks of size $k$, with concurrence graph $G$ and Levi graph $\tilde{G}$. The following statements are equivalent.

(i) The design d is D-optimal.

(ii) The graph $G$ has the maximal number of spanning trees among concurrence graphs of such designs.

(iii) The graph $\tilde{G}$ has the maximal number of spanning trees among Levi graphs of such designs.

Cheng used this theorem in $[10,11]$ with the concurrence graph to find some Doptimal designs whose concurrence matrices are highly patterned. Gaffke used it in [16] with both graphs to find D-optimal designs for $v \leq 6$. Bapat and Dey used it in [8] with the Levi graph to show that all connected designs are D-optimal when $c=0$. Balasubramanian and Dey extended this in [7] to find all D-optimal designs when $c=1$ and $c=2$, but unfortunately gave the wrong constant of proportionality in Sachs' theorem.

\subsection{Electrical Networks}

Denote by $V_{i j}$ the variance of the best linear unbiased estimator of the difference between treatments $i$ and $j$ when the variance of each observation is unity. Thus

$$
V_{i j}=C_{i i}^{-}+C_{j j}^{-}-2 C_{i j}^{-}
$$

where $C_{i j}^{-}$is the $(i, j)$ entry of the Moore-Penrose generalized inverse $\mathbf{C}^{-}$of $\mathbf{C}$. Paterson and Wild used concurrence graphs to evaluate block designs in $[26,27,35]$, using a heuristic argument that, in general terms, $V_{i j}$ should increase according to the distance between vertices $i$ and $j$ in the graph $G$, where distance is the length of the shortest path connecting the two vertices. This is true for some nicely patterned graphs, but is not true in all cases, as shown in [2].

When microarray experiments became popular, there was a sudden need for Aoptimal block designs with $k=2$. The designs were drawn as their concurrence graphs. Jones and Eccleston [19], Kerr and Churchill [20] and Wit et al. [36] found A-optimal designs for small values of $v$ and $b$ by computer search. In [1], Bailey gave analytical proofs of these results, and extended them to larger values. In [3], Bailey and Cameron observed that $V_{i j}$ is proportional, not to distance in $G$, but to resistance-distance in $G$, which is explained below. Tjur had already proved in [33] that $V_{i j}$ is equal to resistance-distance in the Levi graph $\tilde{G}$, which he called the design network. He said "It is tempting to ask ... whether it makes sense to think of the variety-concurrence graph as an electrical network" and concluded that it does.

Any connected graph $H$ can be considered as an electrical network with a 1-ohm resistance in each edge. Suppose that a battery is connected to vertices $i$ and $j$, where 
$i \neq j$, giving a potential difference of 1 volt between those vertices. Then current flows in the network according to the three standard laws of electrical networks.

Ohm's Law: In every edge, the (signed) current is equal to the (signed) potential difference between the vertices at its ends.

Kirchhoff's voltage law: The total (signed) potential difference around any cycle is zero.

Kirchhoff's current law: At every vertex except $i$ and $j$, the total (signed) outgoing current is zero.

These laws give a unique solution for the total current between vertices $i$ and $j$. The reciprocal of its absolute value is called the effective resistance or resistance distance between vertices $i$ and $j$. Electrical network theory shows that this effective resistance is equal to $L_{i i}^{-}+L_{j j}^{-}-2 L_{i j}^{-}$, where $L_{i j}^{-}$is the $(i, j)$ entry of the Moore-Penrose generalized inverse of $\mathbf{L}(H)$ : see [6]. Thus results of [3,4,33,34] can be summarized as follows.

Theorem 4 Let $i$ and $j$ be distinct treatments in an incomplete-block design with block size $k$. Let $R_{i j}$ and $\tilde{R}_{i j}$ be the effective resistances between $i$ and $j$ in the graphs $G$ and $\tilde{G}$, respectively. Then $V_{i j}=\tilde{R}_{i j}=k R_{i j}$.

This result gives an efficient method for calculating the values $V_{i j}$ when the graph does not have many edges. Moreover, it gives the following analogue of Theorem 3.

Theorem 5 Consider a connected incomplete-block design $d$ for $v$ treatments in $b$ blocks of size $k$, with concurrence graph $G$ and Levi graph $\tilde{G}$. The following statements are equivalent.

(i) The design d is A-optimal.

(ii) Among concurrence graphs of such designs, the graph $G$ minimizes the sum of the resistance distances between pairs of distinct vertices.

(iii) Among Levi graphs of such designs, the graph $\tilde{G}$ minimizes the sum of the resistance distances between pairs of distinct treatment-vertices.

For example, consider the resistance distance between treatment-vertices 1 and 3 in Fig. 2. The anticlockwise route around the cycle from 1 to 3 has eight edges, so, if a current of one amp goes on this route from vertex 1 to vertex 3 , then the voltage difference between those two vertices is equal to eight volts. Then current will also flow along the shorter, clockwise route. This has four edges, and a voltage difference of eight volts, so the current must be two amps. Therefore the total current is three amps. The definition of effective resistance assumes a potential difference of 1 volt, so we scale so that the voltage difference is just one volt. This makes the current $3 / 8$, and so $\tilde{R}_{12}=8 / 3$.

This example demonstrates the two key methods of combining resistance distances, which are explained in [6]. If two paths have a single vertex in common, then their union gives a single path: the resistance distance between its endpoints is the sum of the resistance distances of the original paths. This is known as combining resistances in series. If the two paths have the same endpoints but no other vertices in common, then their resistance distances are in parallel: to combine these, we add the reciprocals of both, and then invert that. 


\subsection{Which Graph to Use?}

When $k=2$, the concurrence graph is effectively the same as the design, and it seems natural to use it to calculate the number of spanning trees and the pairwise effective resistances. When $k=3$, both graphs have $3 b$ edges, but the Levi graph has more vertices, so again it is simpler to use the concurrence graph. For larger values of $k$, the Levi graph has fewer edges, and the concurrence graph has $b$ complete subgraphs of size $k$, so that calculations become simpler in the Levi graph. For theorems valid for all values of $k$, it seems simpler to use the Levi graph, although visualizing the graph for a given design with small $k$ is easier with the concurrence graph. If $b>v-1$, then Theorem 2 shows that the Levi graph has more spanning trees and so it may be easier to count them in the concurrence graph; if $b<v-1$, then it is the other way around.

The remaining sections of the paper prove some general results valid for all values of $k$, so the proofs are given using the Levi graph in all cases.

\section{Bridges and Cut-Vertices}

\subsection{Definitions}

One way of measuring the connectivity of a graph is to find out how many edges (or vertices) need to be removed to disconnect the graph.

Definition 9 An edge of a connected graph is a bridge if its removal disconnects the graph. A vertex of a connected graph is a cut-vertex if its removal (together with all edges incident to it) leaves a disconnected graph.

Thus, if a graph is a tree, then every edge is a bridge.

For example, in Fig. 3, vertex 1 is a cut-vertex. The three edges coming down from that vertex are all bridges. Vertex 4 is not a cut-vertex, but the single edge incident with that vertex is a bridge.

A bridge in the Levi graph corresponds to an observation whose loss makes some treatment contrast inestimable. In the terminology of Latif et al. [23], if the Levi graph has a bridge, then the design has breakdown number one.

Lemma 1 Suppose that a connected graph $K$ has a cut-vertex $W$, cutting it into components $H_{1}$ and $H_{2}$ both containing $W$. Let $c, c_{1}$ and $c_{2}$ be the cycle ranks of $K, H_{1}$ and $\mathrm{H}_{2}$, respectively. Then $c=c_{1}+c_{2}$.

Proof For $m=1,2$, suppose that $H_{m}$ contains $e_{m}$ edges and $v_{m}$ vertices in addition to $W$. See Fig. 7. Then $c_{m}=e_{m}-v_{m}$ for $m=1,2$. The graph $K$ has $e_{1}+e_{2}$ edges and $v_{1}+v_{2}+1$ vertices, and so $c=e_{1}+e_{2}-v_{1}-v_{2}=c_{1}+c_{2}$.

In the rest of this section, we show that optimality places strong conditions on the potential occurrences of bridges and cut-vertices in the Levi graph. 


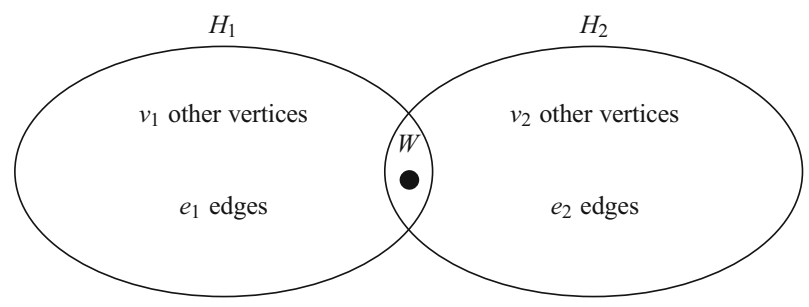

Fig. 7 Graph in the proof of Lemma 1: $W$ is a cut-vertex

\subsection{General Results for D-optimality}

In [5], Bailey, Schiffl and Hilgers proved the following.

Theorem 6 If $G$ is the concurrence graph of a D-optimal block design with $k=2$, $v \geq 3$ and $c \geq 1$, then $G$ does not contain a bridge.

When $k=2$, the Levi graph has a bridge if and only if the concurrence graph has a bridge, so Theorem 6 can be rephrased in terms of the Levi graph. Here we prove a stronger result.

Theorem 7 Let $\tilde{G}$ be the Levi graph of a D-optimal block design $d$ with $c \geq 1$. Then $\tilde{G}$ does not contain any bridge, except, possibly, a bridge whose treatment-vertex has degree 1 .

Proof Since $c \geq 1, \tilde{G}$ is not a tree, so, if it does contain a bridge, then it contains a bridge $e$ joining treatment-vertex $i$ to block-vertex $\Gamma$ in which at least one of those two vertices is contained in a cycle of $\tilde{G}$. If $e$ is removed, then $\tilde{G}$ splits into two connected components. Denote by $H$ and $K$ the components containing $i$ and $\Gamma$, respectively. There are no other edges between $H$ and $K$.

First, suppose that $i$ is contained in a cycle in $H$, and that $f$ is one edge of this cycle, joining treatment-vertex $i$ to block-vertex $\Delta$. See Fig. 8. Let $n_{1}$ be the number of spanning trees of $H$ which contain $f$, and let $n_{2}$ be the number of spanning trees of $H$ which do not contain $f$. Because $f$ is in a cycle, $n_{2}>0$. Let $m$ be the number of spanning trees of $K$. The block-vertex $\Gamma$ has degree $k$, so it has $k-1$ edges in $K$, so $K$ has more than one vertex. Therefore, because $K$ is connected, $m>0$. Every spanning tree of $\tilde{G}$ consists of a spanning tree for $H$, the bridge $e$, and a spanning tree for $K$. Therefore $\tilde{G}$ has $\left(n_{1}+n_{2}\right) m$ spanning trees, where $n_{2}>0$.

Let $j$ be one of the treatment-vertices in $K$ which are joined to block-vertex $\Gamma$. Create a new design $d^{*}$ from $d$ by removing $i$ from $\Delta$ and replacing it by $j$, so that all blocks still have size $k$. Let $\tilde{G}^{*}$ be the Levi graph of $d^{*}$, which omits the edge $f$ but contains the edge $e^{\prime}$ between $j$ and $\Delta$. Let $T$ and $T^{\prime}$ be spanning trees for $H$ and $K$ respectively. If $T$ does not contain $f$, then $T \cup\{e\} \cup T^{\prime}$ and $T \cup\left\{e^{\prime}\right\} \cup T^{\prime}$ are both spanning trees for $\tilde{G}^{*}$; if $T$ does contain $f$, then $(T \backslash\{f\}) \cup\left\{e, e^{\prime}\right\} \cup T^{\prime}$ is a spanning tree for $\tilde{G}^{*}$. Therefore the number of spanning trees for $\tilde{G}^{*}$ is at least $\left(n_{1}+2 n_{2}\right) m$, which is strictly greater than $\left(n_{1}+n_{2}\right) m$ because $n_{2}>0$ and $m>0$. This contradicts the D-optimality of design $d$. 


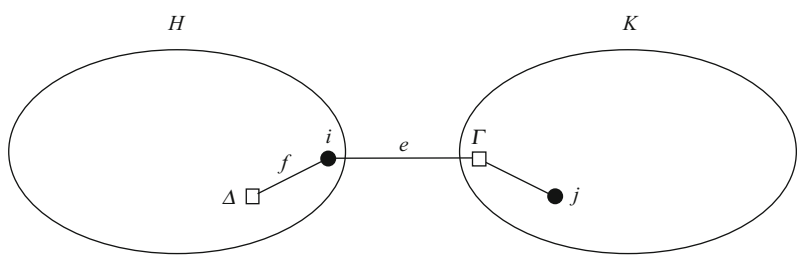

Fig. 8 Graph in the first case in the proof of Theorem 7: $e$ is a bridge

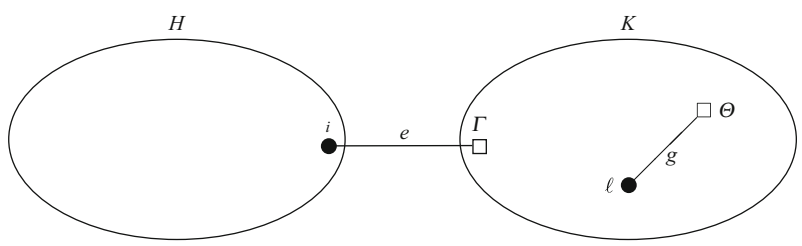

Fig. 9 Graph in the second case in the proof of Theorem 7: $e$ is a bridge

Secondly, suppose that $\Gamma$ is contained in a cycle in $K$. The argument is similar, but with some modifications, so we give it in full. Let $g$ be one edge in this cycle, joining treatment-vertex $\ell$ to block-vertex $\Theta$ (which may or may not be the same as $\Gamma$ ). See Fig. 9. Let $m_{1}$ be the number of spanning trees of $K$ which contain $g$, and let $m_{2}$ be the number of spanning trees of $K$ which do not contain $g$. Then $m_{2}>0$. Let $n$ be the number of spanning trees of $H$. If $\operatorname{deg}(i)>1$, then $H$ has more than one vertex and so $n>0$; if $\operatorname{deg}(i)=1$, then $H$ consists of the single vertex $i$, which by itself forms the only spanning tree for $H$, and so $n=1$. In both cases, the number of spanning trees of $\tilde{G}$ is $\left(m_{1}+m_{2}\right) n$.

Create a new design $d^{* *}$ from $d$ by removing $\ell$ from $\Theta$ and replacing it by $i$; all blocks still have size $k$. The Levi graph $\tilde{G}^{* *}$ of $d^{* *}$ is obtained from $\tilde{G}$ by removing the edge $g$ and inserting an edge $e^{\prime \prime}$ between $i$ and $\Theta$. Let $T$ and $T^{\prime}$ be spanning trees for $H$ and $K$ respectively. If $T^{\prime}$ does not contain $g$, then $T \cup\{e\} \cup T^{\prime}$ and $T \cup\left\{e^{\prime \prime}\right\} \cup T^{\prime}$ are both spanning trees for $\tilde{G}^{* *}$; if $T^{\prime}$ does contain $g$, then $T \cup\left\{e, e^{\prime \prime}\right\} \cup\left(T^{\prime} \backslash\{g\}\right)$ is a spanning tree for $\tilde{G}^{* *}$. Therefore the number of spanning trees for $\tilde{G}^{* *}$ is at least $\left(m_{1}+2 m_{2}\right) n$, which is strictly greater than $\left(m_{1}+m_{2}\right) n$ as $m_{2}>0$ and $n>0$, again contradicting the D-optimality of design $d$.

As an example of the proof of Theorem 7, consider the block design in Fig. 1b. Its Levi graph is in Fig. 3. This has a single cycle, which has length 6, so it has 6 spanning trees. Let $e$ be the bridge joining treatment-vertex 1 to the vertex for the block $\Gamma$ containing treatment 18 , and let $f$ be the edge joining treatment-vertex 1 to the block $\Delta$ containing treatment 2 . Create the new design by removing treatment 1 from $\Delta$ and replacing it by treatment 18 . The new design has a single cycle of length 8 , containing the vertices for treatments 2, 3,1 and 18 in order, so it has 8 spanning trees.

Note that, if $b k<2 v$, then the design must have at least one treatment $i$ with $r_{i}=1$ and so even a D-optimal design will have a bridge in which one of its vertices has degree 1 . A vertex with degree 1 is called a leaf. Let us call a bridge leafless if neither of its vertices is a leaf. 


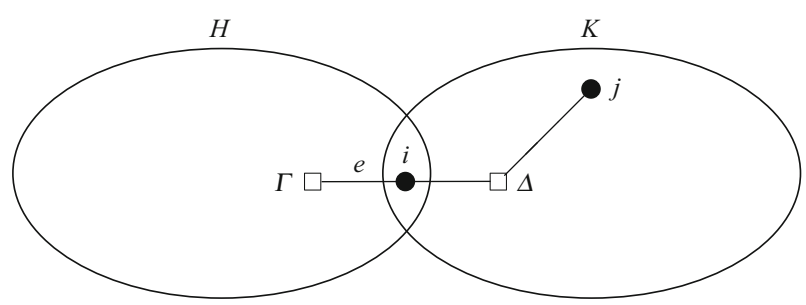

Fig. 10 Graph in the proof of Theorem 8: $i$ is a cut-vertex

Theorem 8 If $\tilde{G}$ is the Levi graph of a D-optimal block design $d$ with $c \geq 1$, then no treatment-vertex of $\tilde{G}$ is a cut-vertex.

Proof Suppose that treatment-vertex $i$ is a cut-vertex which splits $\tilde{G}$ into components $H$ and $K$ both containing $i$ as well as other vertices. Thus $\operatorname{deg}(i) \geq 2$ and there are no edges between $H$ and $K$ except those through vertex $i$. Let $e$ be an edge through $i$ in $H$, joining $i$ to block-vertex $\Gamma$. Since $c \geq 1$, Theorem 7 shows that $e$ is not a bridge. Therefore $e$ is contained in a cycle in $H$. Hence there is at least one spanning tree for $H$ which contains $e$, and at least one which omits it. See Fig. 10.

Suppose that $i$ is joined to block-vertex $\Delta$ in $K$. Because $\operatorname{deg}(\Delta) \geq 2$, there is a treatment-vertex $j$ joined to $\Delta$ with $j \neq i$. Create a new design $d^{*}$ from $d$ by removing $i$ from $\Gamma$ and replacing it by $j$. The Levi graph $\tilde{G}^{*}$ of $d^{*}$ is obtained from $\tilde{G}$ by replacing the edge $e$ with an edge $f$ connecting $j$ to $\Gamma$.

Every spanning tree of $\tilde{G}$ has the form $T \cup T^{\prime}$, where $T$ and $T^{\prime}$ are spanning trees for $H$ and $K$ respectively. Thus the number of spanning trees for $\tilde{G}$ is $n_{1} n_{2}$, where $n_{1}$ and $n_{2}$ are the number of spanning trees for $H$ and $K$ respectively. If $T$ contains $e$, then $(T \backslash\{e\}) \cup\{f\} \cup T^{\prime}$ is a spanning tree for $\tilde{G}^{*}$. If $T$ does not contain $e$, then $T \cup T^{\prime}$ is a spanning tree for $\tilde{G}^{*}$. Furthermore, $T^{\prime}$ contains a unique path from $i$ to $j$, and this has positive even length (it may not be the path through vertex $\Delta$ shown in Fig. 10, because $T^{\prime}$ does not contain all edges in $K$ ). If $e^{\prime}$ is any one of the edges in this path, then $T \cup\{f\} \cup\left(T^{\prime} \backslash\left\{e^{\prime}\right\}\right)$ is a spanning tree for $\tilde{G}^{*}$. Different choices for $T^{\prime}$ may give rise to the same spanning tree of this type. Nevertheless, at least two of them are distinct, and so the number of spanning trees for $\tilde{G}^{*}$ is at least $n_{1} n_{2}+2$, which contradicts the D-optimality of $d$.

\subsection{General Results for A-optimality}

Theorem 9 Suppose that $i$ is a treatment in a block design $d$ and that its treatmentvertex is a cut-vertex of the Levi graph $\tilde{G}$ of d, cutting it into components $\mathrm{H}_{1}$ and $\mathrm{H}_{2}$ both containing $i$. For $m=1$, 2, let $H_{m}$ contain $v_{m}$ treatment-vertices in addition to $i$, let $R_{m}$ be the sum of the resistance distances between pairs of treatment-vertices in $H_{m}$ and let $S_{m}$ be the sum of these in $H_{m}$ which involve treatment-vertex $i$. Let $K_{m}$ be the set of treatment-vertices, including $i$, in $H_{m}$. Thus $S_{m}=\sum_{j \in K_{m}} \tilde{R}_{i j}$, because $\tilde{R}_{i i}=0$, and $R_{m}=\left(\sum_{j \in K_{m}} \sum_{\ell \in K_{m}} R_{j \ell}\right) / 2$, because $\tilde{R}_{j j}=0$ and $\tilde{R}_{j \ell}=\tilde{R}_{\ell j}$ for all 


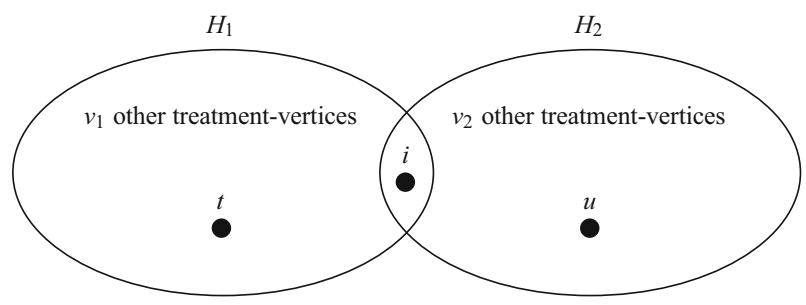

Fig. 11 Graph in the proof of Theorem 9: $i$ is a cut-vertex

$j$ and $\ell$. Then the sum of the resistance distances between pairs of treatment-vertices in $\tilde{G}$ is $R_{1}+R_{2}+v_{2} S_{1}+v_{1} S_{2}$.

Proof See Fig. 11. All resistance distances involving treatment-vertex $i$ within $H_{1}$ or $H_{2}$ are included in $R_{1}+R_{2}$. If $t$ is a treatment-vertex in $K_{1} \backslash\{i\}$ and $u$ is a treatmentvertex in $K_{2} \backslash\{i\}$, then $\tilde{R}_{t u}=\tilde{R}_{t i}+\tilde{R}_{i u}$. Summing this over all $t$ and $u$ gives $v_{2} S_{1}+v_{1} S_{2}$, and the result follows.

The following lemma shows the use of Theorem 9 on a small example. Not only does this help in understanding Theorem 9; it will also be used in the proof of Theorem 11.

Lemma 2 In addition to the assumptions and notation in Theorem 9, suppose that $k=3$ and $v_{1}=4$. Suppose that component $H_{1}$ is as shown in Fig. 12a, so that block $\Delta$ contains treatments $i, t$ and $u$, while block $\Gamma$ contains treatment $j$ twice and treatment $i$ once. Then $d$ is not A-optimal.

Proof In $H_{1}$, the resistance distances between pairs of treatment-vertices are

$$
\tilde{R}_{i j}=3 / 2, \quad \tilde{R}_{i t}=\tilde{R}_{i u}=\tilde{R}_{t u}=2 \text { and } \tilde{R}_{j t}=\tilde{R}_{j u}=7 / 2 .
$$

Hence $R_{1}=29 / 2$ and $S_{1}=11 / 2$.

Construct a new design $d^{*}$ from $d$ by removing one occurrence of $j$ from $\Gamma$, inserting it into $\Delta$, and removing $t$ from $\Delta$ and inserting it into $\Gamma$, thus giving $H_{1}^{*}$ in Fig. 12b. The resistance distances between pairs of treatment-vertices in $H_{1}^{*}$ are

$$
\tilde{R}_{i j}^{*}=1, \quad \tilde{R}_{i t}^{*}=\tilde{R}_{i u}^{*}=\tilde{R}_{j t}^{*}=\tilde{R}_{i u}^{*}=7 / 4 \text { and } \tilde{R}_{t u}^{*}=3 .
$$

Hence $R_{1}^{*}=11<R_{1}$ and $S_{1}^{*}=9 / 2<S_{1}$.

Theorem 9 shows that the sum of the resistance distances between pairs of treatmentvertices in the Levi graph is

$$
\begin{array}{ll}
R_{1}+R_{2}+v_{2} S_{1}+4 S_{2} & \text { for design } d, \text { and } \\
R_{1}^{*}+R_{2}+v_{2} S_{1}^{*}+4 S_{2} & \text { for design } d^{*} .
\end{array}
$$

Since $R_{1}^{*}<R_{1}$ and $S_{1}^{*}<S_{1}$, the second value is strictly smaller, regardless of the properties of $\mathrm{H}_{2}$. By Theorem 5, the design $d$ cannot be A-optimal. 


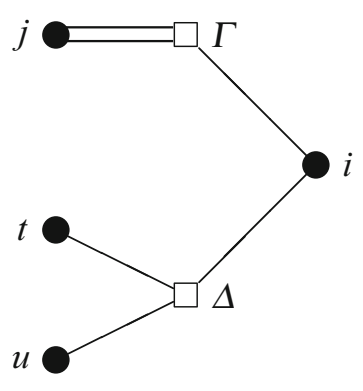

(a) $H_{1}$

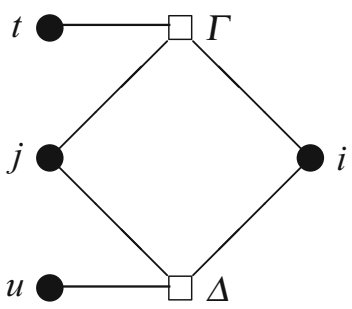

(b) $H_{1}^{*}$

Fig. 12 Two possibilities for one component of the Levi graph in Lemma 2

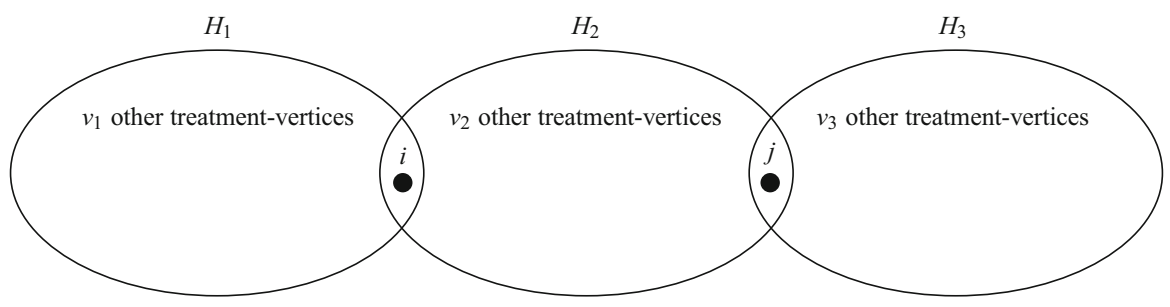

Fig. 13 Graph in the proof of Theorem 10: $i$ and $j$ are both cut-vertices

Theorem 10 Let $\tilde{G}$ be the Levi graph of an A-optimal block design d. Then there cannot be two distinct treatments $i$ and $j$ whose vertices are both cut-vertices.

Proof Suppose that there are two such treatments $i$ and $j$. Then removing vertices $i$ and $j$ splits the graph $\tilde{G}$ into three components $H_{1}, H_{2}$ and $H_{3}$, each with at least two vertices, where treatment-vertex $i$ is the only vertex in $H_{1} \cap H_{2}$, treatment-vertex $j$ is the only vertex in $H_{2} \cap H_{3}$ and $H_{1} \cap H_{3}$ contains no vertices. Each component must be connected, because the whole Levi graph is connected. For $\ell \in\{1,2,3\}$, let $K_{\ell}$ be the set of treatment-vertices in $H_{\ell}$, and let $v_{\ell}=\left|K_{\ell} \backslash\{i, j\}\right|$. See Fig. 13.

Let

$$
S_{1}=\sum_{t \in K_{1}} \tilde{R}_{i t}, \quad S_{2}=\sum_{t \in K_{2}} \tilde{R}_{i t}, \quad S_{3}=\sum_{t \in K_{2}} \tilde{R}_{j t} \quad \text { and } \quad S_{4}=\sum_{t \in K_{3}} \tilde{R}_{j t} .
$$

Then

$$
\sum_{t \in K_{1}} \tilde{R}_{j t}=S_{1}+\left(v_{1}+1\right) \tilde{R}_{i j} \quad \text { and so } \sum_{t \in K_{1} \cup K_{2}} \tilde{R}_{j t}=S_{3}+S_{1}+v_{1} \tilde{R}_{i j}
$$

(after removing the double occurrence of $\tilde{R}_{i j}$, because $K_{1} \cap K_{2}=\{i\}$ ).

Let $R_{0}$ and $R_{3}$ be the sums of the resistance distances between pairs of treatmentvertices in $K_{1} \cup K_{2}$ and $K_{3}$, respectively. Theorem 9 shows that the sum of the resistance distances between pairs of treatment-vertices in $\tilde{G}$ is 


$$
R_{0}+R_{3}+\left(v_{1}+v_{2}+1\right) S_{4}+v_{3}\left(S_{1}+S_{3}+v_{1} \tilde{R}_{i j}\right)
$$

There is no loss of generality in assuming that $S_{2} \leq S_{3}$. Create design $d^{*}$ from $d$ by replacing treatment $j$ by treatment $i$ in all those blocks containing $j$ whose other treatment-vertices are in $K_{3}$. Let $\tilde{G}^{*}$ be the Levi graph of $d^{*}$, and denote resistance distances in this graph by $\tilde{R}^{*}$. Then

$$
\sum_{t \in K_{1} \cup K_{2}} \tilde{R}_{i t}^{*}=S_{1}+S_{2}
$$

and so Theorem 9 shows that the sum of the resistance distances between pairs of treatment-vertices in $\tilde{G}^{*}$ is

$$
R_{0}+R_{3}+\left(v_{1}+v_{2}+1\right) S_{4}+v_{3}\left(S_{1}+S_{2}\right)
$$

Since $S_{2} \leq S_{3}$ and $0<v_{1} v_{3} \tilde{R}_{i j}$, the comparison of expressions (1) and (2) shows that the sum of the resistance distances between pairs of treatment-vertices in $\tilde{G}^{*}$ is strictly less than the corresponding sum in $\tilde{G}$, which contradicts the A-optimality of $d$, by Theorem 5 .

Theorem 11 If $\tilde{G}$ is the Levi graph of an A-optimal block design $d$ with $c \geq 1$, then no treatment-vertex of $\tilde{G}$ is a cut-vertex unless it is contained in a cycle.

Proof Suppose that treatment $i$ gives such a cut-vertex, contained in no cycle and cutting $\tilde{G}$ into components $H_{1}$ and $H_{2}$ both containing $i$. Without loss of generality, there is a cycle in $H_{1}$. If $H_{1} \backslash\{i\}$ is not connected then we may move any, but not all, of its connected components into $\mathrm{H}_{2}$ without affecting the assumptions, so long as we leave a cycle in $H_{1}$. Thus we may assume that $H_{1} \backslash\{i\}$ is connected.

Suppose that treatment $i$ is in blocks $\Gamma_{1}$ and $\Gamma_{2}$ whose vertices are in $H_{1}$. Since $H_{1} \backslash\{i\}$ is connected, it contains a path from $\Gamma_{1}$ to $\Gamma_{2}$. Adding the edges from $\Gamma_{1}$ and $\Gamma_{2}$ to $i$ gives a cycle containing vertex $i$. This is contradicts our assumption that $i$ is not in a cycle.

Otherwise, suppose that treatment $i$ is in a unique block $\Gamma$ in $H_{1}$. Because $H_{1}$ is connected and contains a cycle, there is at least one treatment-vertex $j$ in $H_{1}$ that is joined to $\Gamma$ and is not a leaf. See Fig. 14. If the edge between $\Gamma$ and $j$ is not in a cycle, then $j$ is also a cut-vertex, which contradicts Theorem 10. Hence this edge is in a cycle. If the cycle has length $2 n$, then the rule for combining resistance distances in parallel given in Sect. 3.2 shows that

$$
\tilde{R}_{j \Gamma} \leq\left[1+\frac{1}{2 n-1}\right]^{-1}=\frac{2 n-1}{2 n}<1 .
$$

On the other hand, $\tilde{R}_{i \Gamma}=1$ because the edge between $\Gamma$ and $i$ is not contained in any cycle. In particular, there cannot be multiple edges between $\Gamma$ and $i$.

For $m=1,2$, let $R_{m}$ be the sum of the resistance distances between pairs of treatment-vertices in $H_{m}$, and let $v_{m}$ be the number of treatment-vertices in $H_{m}$ in 


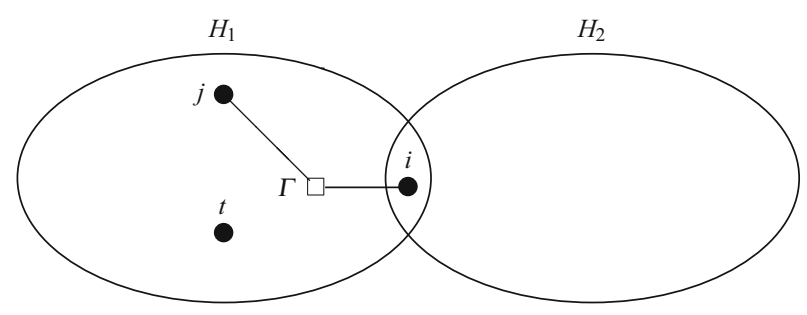

Fig. 14 Graph in the proof of Theorem 11: $j$ is a cut-vertex not contained in a cycle

addition to $i$. Let $S_{i}$ and $S_{j}$ be the sum of resistance distances between treatmentvertices in $H_{1}$ and vertices $i$ and $j$, respectively, and let $S$ be the sum of resistance distances between vertex $i$ and treatment-vertices in $H_{2}$. Theorem 9 shows that the sum of the resistance distances for pairs of treatment-vertices in $\tilde{G}$ is $R_{1}+R_{2}+v_{2} S_{i}+v_{1} S$.

Let $t$ be a treatment, other than $i$ and $j$, whose vertex is in $H_{1}$. Because resistance distance is a metric,

$$
\tilde{R}_{j t} \leq \tilde{R}_{j \Gamma}+\tilde{R}_{t \Gamma}<1+\tilde{R}_{t \Gamma}=\tilde{R}_{i t}
$$

Hence $S_{j}<S_{i}$.

Create a new design $d^{*}$ from $d$ by replacing treatment $i$ with treatment $j$ in every block containing $i$ whose vertex is in $H_{2}$. Then the sum of the resistance distances for pairs of treatment-vertices in the new Levi graph is $R_{1}+R_{2}+v_{2} S_{j}+v_{1} S$, which is strictly less than it was in $\tilde{G}$. This contradicts the A-optimality of $d$, by Theorem 5 .

The last step in the proof breaks down if $i$ and $j$ are the only treatment-vertices in $H_{1}$. In this case, $j$ is joined to $\Gamma$ by at least two edges. As noted above, there is only one edge between $i$ and $\Gamma$. Hence there are $k-1$ edges between $j$ and $\Gamma$. Thus $\tilde{R}_{i j}=1+1 /(k-1)=k /(k-1)$. If $k \geq 4$, then replacing one occurrence of treatment $j$ in block $\Gamma$ by a second copy of treatment $i$ reduces $\tilde{R}_{i j}$ to $1 / 2+1 /(k-2)=k /(2 k-4)$. This reduces all the resistance distances from $j$ to treatment-vertices within $H_{2}$, while leaving all those within $\mathrm{H}_{2}$ unchanged. Thus $d$ cannot be A-optimal, by Theorem 5 .

The only remaining possibility is that $k=3$ and that vertex $i$ cuts $\tilde{G}$ into connected components, each containing one block. At least one block contains two copies of one treatment, which is not $i$. The remaining blocks are either like this or contain three distinct treatments. Hence there are either a pair of components giving the graph in Fig. 12a or there are a pair giving the graph in Fig. 15a. In the first case, Lemma 2

Fig. 15 Two possibilities for one component of the Levi graph at the end of the proof of Theorem 11

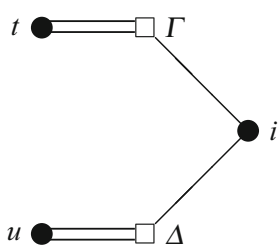

(a)

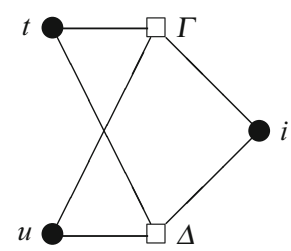

(b) 
shows that $d$ is not A-optimal. In the second case, a similar argument shows that replacing the graph in Fig. 15a by the one in Fig. 15b, while leaving the rest of the design unchanged, gives a design with a strictly smaller sum of resistance distances between pairs of treatment-vertices, and so $d$ is not A-optimal, by Theorem 5 .

\section{D-optimal Block Designs with Low Cycle Rank}

\subsection{Minimal Number of Observations}

Theorem 12 If $b k=b+v-1$, then all connected block designs for $v$ treatments in $b$ blocks of size $k$ are D-optimal.

Proof In this case, the number of edges of the Levi graph is one less than its number of vertices. Hence, if it is connected, then it must be a tree, and so it has precisely one spanning tree. If it is not connected, it has none.

Theorem 12 was proved in $[8,25]$. We include it here for completeness.

\subsection{Nearly Minimal Number of Observations}

Since $c=b k-b-v+1, c=1$ if and only if $v=b(k-1)$. Thus there is a single cycle if and only if $v=b(k-1)$. In this case, $b=v /(k-1) \leq v$ and $\operatorname{so} \min \{b, v\}=b$.

Theorem 13 If $b k=b+v$, then every D-optimal design is obtainable from the following by relabelling treatments and relabelling blocks. For $i=1, \ldots, b-1$, block $\Gamma_{i}$ contains treatments $i$ and $i+1$, while block $\Gamma_{b}$ contains treatments $b$ and $1 . A$ further $k-2$ treatments, all with replication 1 , are inserted into each block.

Proof In this case, the Levi graph contains a single cycle. Each spanning tree is obtained by removing one edge from the cycle, so the design is D-optimal if and only if the cycle has maximal length, which is $\min \{v, b\}=b$.

For example, this theorem shows that the block design in Fig. 1a is D-optimal.

Theorem 13 was proved in [7]. Again, we include it both for completeness and to show the benefit of the unified approach.

\subsection{One More Observation}

If $b k=b+v+1$, then $c=2$. Let $\tilde{G}$ be the Levi graph of a block design $d$ for $v$ treatments in $b$ blocks of size $k$. When $c=2$, then there are only three possibilities for how the cycles occur in a graph: (a) there are two separate cycles, with no vertices in common; (b) there are two cycles with a single vertex in common; (c) there are two special vertices joined by three distinct paths.

In case (a), connectivity implies that there is a path connecting the two cycles. Every edge of this path is a leafless bridge, so Theorem 7 shows that $d$ is not D-optimal. 
In case (b), the single vertex in common to the two cycles is a cut-vertex. If $d$ is D-optimal, then Theorem 8 shows that no treatment-vertex is a cut-vertex of $\tilde{G}$. So suppose that $\Gamma$ is a block whose vertex is a cut-vertex of $\tilde{G}$, cutting it into components $H_{1}$ and $H_{2}$ both containing $\Gamma$. Suppose further that both $H_{1}$ and $H_{2}$ contain other block-vertices in addition to $\Gamma$. Theorem 7 shows that neither $H_{1}$ nor $H_{2}$ contains a leafless bridge, so all of their block-vertices are contained in cycles. By Lemma 1 , each component has a single cycle containing all its block-vertices. Figure 16 shows an example, excluding the treatment-vertices outside the cycles. For $m=1,2$, let $b_{m}$ be the number of block-vertices in $H_{m}$ in addition to $\Gamma$. Thus $b=b_{1}+b_{2}+1 \geq 3$, and $H_{m}$ contains $2\left(b_{m}+1\right)$ spanning trees, each made by omitting one edge from its cycle. Thus the number of spanning trees of $\tilde{G}$ is $4\left(b_{1}+1\right)\left(b_{2}+1\right)$. This is maximized when $b_{1}$ and $b_{2}$ are as equal as possible. If $b=2 n+1$, then it is maximized at $(b+1)^{2}$ when $b_{1}=b_{2}=n$; if $b=2 n+2$, then it is maximized at $b(b+2)$ when $b_{1}$ and $b_{2}$ are equal to $n$ and $n+1$ in either order.

In case (c) there are two special vertices $W_{1}$ and $W_{2}$ in $\tilde{G}$ which are joined by three disjoint paths. Because there are no bridges, every block-vertex is either one of $W_{1}$ and $W_{2}$ or is contained in a single path. If $W_{1}$ and $W_{2}$ are both block-vertices, then the path lengths are all even and sum to $2 b+2$. Figure 17 shows an example, excluding the treatment-vertices outside the cycles. If $W_{1}$ and $W_{2}$ are both treatment-vertices, then the path lengths are all even and sum to $2 b$; otherwise, the path lengths are all odd and sum to $2 b+1$.

Denote these path lengths by $p_{1}, p_{2}$ and $p_{3}$. Then the number of spanning trees in $\tilde{G}$ is $p_{1} p_{2}+p_{1} p_{3}+p_{2} p_{3}$. This is maximized when $p_{1}+p_{2}+p_{3}=2 b+2$, so that $W_{1}$ and $W_{2}$ are both block-vertices, and the path lengths are as equal as possible. If $b=3 m+1$, this happens when two of the path lengths are $2 m+2$ and the other is $2 m$; if $b=3 m+2$, it happens when all of the path lengths are $2 m+2$; if $b=3 m+3$, it happens when two of the path lengths are $2 m+2$ and the other is $2 m+4$. In every case, $p_{1} p_{2}+p_{1} p_{3}+p_{2} p_{3}>(b+1)^{2}$, so a design with a block cut-vertex cannot be D-optimal. A D-optimal design can be constructed by drawing a graph with three paths, of the prescribed lengths, between block-vertices $W_{1}$ and $W_{2}$; inserting one treatment in alternate vertices in each path (and hence in the two blocks on either

Fig. 16 An example of the cycles in the Levi graph in the second case in Sect. 5.3, with $b_{1}=5$ and $b_{2}=4$

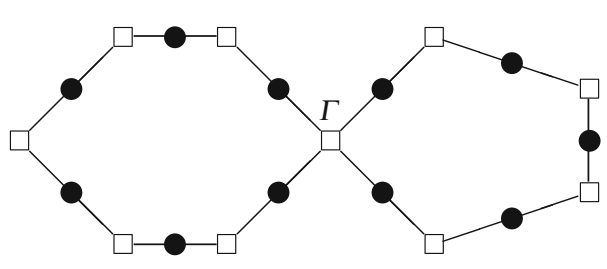

Fig. 17 An example of the cycles of the Levi graph in the third case in Sect. 5.3, with $p_{1}=p_{3}=8$ and $p_{2}=6$

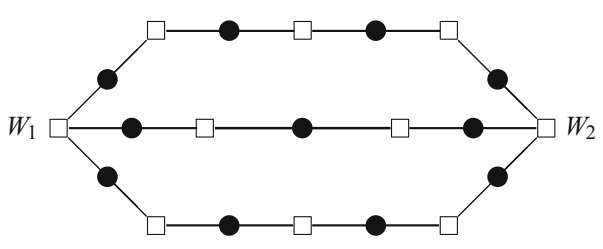


side); inserting $k-3$ other treatments into the blocks with vertices $W_{1}$ and $W_{2}$; and inserting $k-2$ other treatments into every other block. This works whenever $k \geq 3$.

For $k=2$, it is not possible for either of $W_{1}$ or $W_{2}$ to be a block-vertex. A similar argument shows that, in this case, the D-optimal designs are made by allocating two treatments to these vertices, drawing three paths of the prescribed lengths between them, and inserting one block in alternate vertices in each path.

This result was given in [7].

For example, when $b=10, k=4$ and $v=29$, then the non-leaf part of the Levi graph is as shown in Fig. 17. If vertices $W_{1}$ and $W_{2}$ represent blocks 1 and 10, respectively, one possibility for the D-optimal design is shown in Fig. 18. On the other hand, if $b=11, k=2$ and $v=10$, then we need to swap the roles of treatment-vertices and block-vertices in Fig. 17. Figure 19 shows one possibility for the D-optimal design in this case.

\section{A-optimal Block Designs with Low Cycle Rank}

\subsection{Minimal Number of Observations}

Theorem 14 If $b k=b+v-1$, then a block design for $v$ treatments in $b$ blocks of size $k$ is A-optimal if and only if there is one treatment that occurs in every block.

Proof As in the proof of Theorem 12, if the Levi graph is connected, then it must be a tree. Every non-leaf vertex of a tree is a cut-vertex, so Theorem 10 shows that all treatment-vertices but one are leaves, so these treatments have replication 1 . Connectivity then implies that the remaining treatment is in every block. The converse follows from the fact that the Levi graph of each block design that has one treatment that occurs in each block has the same sum of resistance distances.

Designs like those in Theorem 14 are called star designs in [3], and queen-bee designs in [4]. Figure 20 shows an example with $b=8, k=4$ and $v=25$.

Theorem 14 was proved in $[3,8,25]$. Now the groundwork in Sect. 4 makes the proof simpler.

\begin{tabular}{|c|c|c|c|c|c|c|c|c|c|}
\hline 1 & 1 & 2 & 3 & 5 & 6 & 8 & 9 & 10 & 4 \\
5 & 2 & 3 & 4 & 6 & 7 & 9 & 10 & 11 & 7 \\
8 & 13 & 15 & 17 & 19 & 21 & 23 & 25 & 27 & 11 \\
12 & 14 & 16 & 18 & 20 & 22 & 24 & 26 & 28 & 29 \\
\hline
\end{tabular}

Fig. 18 A D-optimal block design with $b=10, k=4$ and $v=29$

\begin{tabular}{|l|l|l|c|c|c|c|c|c|c|c|}
\hline 1 & 2 & 3 & 4 & 1 & 5 & 6 & 1 & 7 & 8 & 9 \\
2 & 3 & 4 & 10 & 5 & 6 & 10 & 7 & 8 & 9 & 10 \\
\hline
\end{tabular}

Fig. 19 A D-optimal block design with $b=11, k=2$ and $v=10$ 


\subsection{Nearly Minimal Number of Observations}

As noted in Sect. 5.2, if $c=1$, then $v=b(k-1)$ and the Levi graph contains a single cycle. Let $s$ be the number of block-vertices in this cycle. Then the cycle contains $s$ treatment-vertices and $2 s$ edges. If $s=b$, then there are no cut-vertices. If $1<s<b$, then there is at least one block-vertex outside the cycle. If the path joining it to the cycle meets the cycle in a block-vertex, then that path contains a treatmentvertex which is not in a cycle and is a cut-vertex: by Theorem 11, the design is not A-optimal. On the other hand, if that path meets the cycle in a treatment-vertex $\ell$, then that vertex is a cut-vertex. Theorem 10 shows that if the block design is A-optimal, then there is no other treatment cut-vertex. Hence treatment-vertex $\ell$ is joined to all the $b-s$ block-vertices outside the cycle. If $s=1$, then the treatment whose vertex is in the cycle occurs twice in the block whose vertex is in the cycle, so connectivity implies that $k \geq 3$. Denote by $\mathscr{C}(b, k, s)$ the set of block designs satisfying these conditions.

Figures 2 and 3 show the Levi graphs of block designs in $\mathscr{C}(6,4,6)$ and $\mathscr{C}(6,4,3)$, respectively.

Lemma 3 Suppose that $v=b(k-1)$ and a block design is in $\mathscr{C}(b, k, s)$. Then the sum of all the pairwise resistance distances between treatment-vertices in the Levi graph is $g(s) / 6$, where

$$
g(s)=-(k-1)^{2} s^{3}+2 b(k-1)^{2} s^{2}-\left[6 b k(k-1)-4 k^{2}+2 k-1\right] s+C,
$$

and $C=b(k-1)[12 b(k-1)-5 k-4]$.

Proof Let $\mathscr{V}_{1}$ be the set of treatment-vertices in the cycle, let $\mathscr{V} 2$ be the set of other treatment-vertices joined to block-vertices in the cycle, and let $\mathscr{V} 3$ be the set of remaining treatment-vertices. Suppose that $i$ and $j$ are in $\mathscr{V}_{1}$, with $i \neq j$. The two paths from $i$ to $j$ have lengths $2 p$ and $2(s-p)$ for some $p$ with $1 \leq p \leq s-1$. The rule for combining resistance distances in parallel gives

$$
R_{i j}=\left(\frac{1}{2 p}+\frac{1}{2(s-p)}\right)^{-1}=\frac{2 p(s-p)}{s}
$$

For each fixed $i$, the sum of $R_{i j}$ as $j$ varies over $\mathscr{V}_{1}$ is

$$
\sum_{p=1}^{s-1} \frac{2 p(s-p)}{s}=\frac{(s-1)(s+1)}{3} .
$$

\begin{tabular}{|c|c|c|c|c|c|c|c|}
\hline 1 & 1 & 1 & 1 & 1 & 1 & 1 & 1 \\
2 & 5 & 8 & 11 & 14 & 17 & 20 & 23 \\
3 & 6 & 9 & 12 & 15 & 18 & 21 & 24 \\
4 & 7 & 10 & 13 & 16 & 19 & 22 & 25 \\
\hline
\end{tabular}

Fig. 20 An A-optimal block design with $b=8, k=4$ and $v=25$ 
Call this $P_{1}$. If $\Gamma$ is a block-vertex in the cycle, then the same argument shows that the sum of the resistance distances from $\Gamma$ to other block-vertices in the cycle is also equal to $P_{1}$.

Similarly, if $i$ and $\Gamma$ are a treatment-vertex and block-vertex in the cycle, then the two paths from $i$ to $\Gamma$ have lengths $2 q-1$ and $2 s-2 q+1$ for some $q$ with $1 \leq q \leq s$. For each fixed $i$, the sum of $R_{i}$ as $\Gamma$ varies over these blocks is

$$
\sum_{q=1}^{s} \frac{(2 q-1)(2 s-2 q+1)}{2 s}=\frac{\left(2 s^{2}+1\right)}{6} .
$$

Call this $P_{2}$.

For $1 \leq m \leq n \leq 3$, denote by $\mathscr{R}_{m n}$ the sum of the pairwise resistance distances between vertices in $\mathscr{V}_{m}$ and $\mathscr{V}_{n}$. Thus $\mathscr{R}_{11}=s P_{1} / 2$.

Suppose that vertex $t$ in $\mathscr{V}_{2}$ is joined to block-vertex $\Gamma$. If $i \in \mathscr{V}_{1}$, then the rule for combining resistance distances in series gives $R_{t i}=R_{t \Gamma}+R_{\Gamma i}=1+R_{\Gamma i}$. Summing over $i$ in $\mathscr{V}_{1}$ gives $s+P_{2}$. There are $s(k-2)$ vertices in $\mathscr{V}_{2}$, and so $\mathscr{R}_{12}=s(k-2)\left(s+P_{2}\right)$.

Let $t$ and $u$ be distinct vertices in $\mathscr{V}$. If they are joined to the same block-vertex, then $R_{t u}=2$. There are $s(k-2)(k-3) / 2$ such pairs, so the sum of these resistance distances is $s(k-2)(k-3)$. If they are joined to different block-vertices $\Gamma$ and $\Delta$, then $R_{t u}=2+R_{\Gamma \Delta}$. There are $(k-2)^{2}$ such pairs for each choice of $\Gamma$ and $\Delta$, so the sum of these resistance distances is $(k-2)^{2}\left[s(s-1)+\mathscr{R}_{11}\right]$. Hence $\mathscr{R}_{22}=s(k-2)(k-3)+s(s-1)(k-2)^{2}+s(k-2)^{2} P_{1} / 2$.

If $t \in \mathscr{V}_{3}$ and $u \in \mathscr{V}_{1} \cup \mathscr{V}_{2}$, then $R_{t u}=2+R_{\ell u}$, where $\ell$ is the cut-vertex. Summing over $u$ in $\mathscr{V}_{1}$ gives $2 s+P_{1}$, while summing over $u$ in $\mathscr{V}_{2}$ gives $(k-2)\left(3 s+P_{2}\right)$. There are $(b-s)(k-1)$ vertices in $\mathscr{V}_{3}$, and so $\mathscr{R}_{13}=(b-s)(k-1)\left(2 s+P_{1}\right)$ and $\mathscr{R}_{23}=(b-s)(k-1)(k-2)\left(3 s+P_{2}\right)$.

If different vertices $t$ and $u$ in $\mathscr{V}_{3}$ are in the same block, then $R_{t u}=2$; otherwise, $R_{t u}=4$. Hence $\mathscr{R}_{33}=(b-s)(k-1)(k-2)+2(b-s)(b-s-1)(k-1)^{2}$.

Adding $\mathscr{R}_{11}, \mathscr{R}_{12}, \mathscr{R}_{13}, \mathscr{R}_{22}, \mathscr{R}_{23}$ and $\mathscr{R}_{33}$ shows that the sum of all the pairwise resistance distances between treatment-vertices in the Levi graphs is $g(s) / 6$.

Theorem 15 If $v=b(k-1)$, then a block design for $v$ treatments in b blocks of size $k$ is A-optimal if and only if it is in $\mathscr{C}(b, k, s)$, where

(i) $s=b$ if $2 \leq b \leq 4$, or $b=5$ and $k \leq 5$, or $b=6$ and $k \leq 3$, or $7 \leq b \leq 8$ and $k=2$;

(ii) $s=4$ if $9 \leq b \leq 11$ and $k=2$;

(iii) $s=3$ or $s=4$ if $b=12$ and $k=2$;

(iv) $s=3$ if $b=6$ and $k=4$, or $7 \leq b \leq 11$ and $k=3$, or $b \geq 13$ and $k=2$;

(v) $s=2$ otherwise.

Proof Lemma 3 shows that we need to find the value of $s$, for $s$ in $\{1,2, \ldots, b\}$, which minimizes $g(s)$. First, we note that

$$
\begin{aligned}
g(1)-g(2) & =7(k-1)^{2}-6 b(k-1)^{2}+6 b k(k-1)-4 k^{2}+2 k-1 \\
& =(3 k+6 b-9)(k-1)-3>0,
\end{aligned}
$$


because $b \geq 2$ and $k \geq 2$. Thus designs in $\mathscr{C}(b, k, 1)$ are never A-optimal.

We now take four steps to find the integer $s$ in $[2, b]$ which minimizes $g(s)$.

Step 1 If we regard the polynomial function $g$ as defined on the real numbers, then

$$
g^{\prime}(s)=-3(k-1)^{2} s^{2}+4 b(k-1)^{2} s-6 b k(k-1)+4 k^{2}-2 k+1 .
$$

This is zero when

$$
s=\frac{2 b}{3} \pm \frac{(k-1) \sqrt{\alpha(b, k)}}{3(k-1)^{2}},
$$

where

$$
\alpha(b, k)=4 b^{2}(k-1)^{2}-18 b k(k-1)+12 k^{2}-6 k+3 .
$$

If $\alpha(b, k)<0$, then $g^{\prime}(s)$ is never zero for real values of $s$, and so $g(s)$ decreases monotonically. Thus $g(s)$ is minimized for $s \in\{1,2, \ldots, b\}$ at $s=b$. This happens for all $k$ when $b=2$ or $b=3$, and for $k \leq 14$ when $b=4$. When $b=4$, then $g(3)-g(4)=k^{2}+16 k-20>0$ for all $k \geq 2$, so $g(s)$ is minimized for $s \in\{1,2,3,4\}$ at $g(4)$.

It is also the case that $\alpha(b, k)<0$ if $b=5$ and $k \leq 4$; if $b=6$ and $k \leq 3$; and if $b=7$ and $k=2$. In all of these cases, $g(s)$ is minimized for $s \in\{1,2, \ldots, b\}$ at $s=b$.

From now on, we may assume that $b \geq 5$.

Step 2 Now we compare $g(b)$ with $g(2)$ and $g(3)$, and show that $g(b)$ is larger than at least one of the other two in almost all of the remaining cases. We have

$$
g(b)-g(2)=b^{2}(k-1)[(b-6)(k-1)-6]+b\left(8 k^{2}-7\right)+2 k(b-6)+6 .
$$

If $b=5$, this is $15 k^{2}-102 k+96$, which is positive whenever $k \geq 6$. If $b=6$, it is $12\left(4 k^{2}-18 k+15\right)$, which is positive whenever $k \geq 4$. For all larger values of $b$, it is positive whenever $k \geq 3$.

When $k=2$,

$$
g(b)-g(3)=b^{2}(b-12)+31 b-12 .
$$

This is positive whenever $b \geq 9$.

Thus $g(b)$ does not give the minimum value if $k \geq 6$ and $b \geq 5$, if $k \geq 4$ and $b \geq 6$, if $k \geq 3$ and $b \geq 7$, or if $k=2$ and $b \geq 9$. In all these cases, it suffices to find the real number $s_{0}$ in $(1, b]$ where $g^{\prime}\left(s_{0}\right)=0$; note that $s_{0} \neq 1$, because $g(1)>g(2)$. If $s_{0}<2$, then the minimum for integer $s$ occurs at $s=2$. Otherwise, if $s_{0}$ is not an integer, compare $g\left(s_{1}\right)$ and $g\left(s_{2}\right)$, where $s_{1}$ and $s_{2}$ are the integers on either side of $s_{0}$.

Step 3 If $g(3)>g(2)$, then the overall minimum of $g(s)$ on $[1, b]$ occurs in $[1,3]$, and the minimum value for integer $s$ is at $s=2$. Now,

$$
g(3)-g(2)=(4 b k-10 b-15 k+21)(k-1)+3 \text {. }
$$


This is always negative when $k=2$. However, $g(3)-g(2)>0$ when $k=3$ and $b \geq 12$; when $k=4$ and $b \geq 7$; when $k=5$ and $b \geq 6$; and when $k \geq 6$ and $b \geq 5$. Apart from the situation with $k=2$, this leaves only a small finite number of cases to be checked.

Step 4 When $k=2, g(s)=-s^{3}+2 b s^{2}-(12 b-13) s+C$. Therefore $g(4)-g(3)=$ $2 b-24$. Hence the minimum occurs at $s=3$ if $b \geq 13$. If $b=12$, then $g(3)=g(4)$ and the minimum value for integers $s$ occurs at both points. If $b \leq 11$, then $g(s)$ is minimized for some $s$ in $[4, b]$. From Step 1, the only remaining cases to check are values of $b$ with $8 \leq b \leq 11$.

For example, this theorem shows that the block design in Fig. 1(b) is A-optimal.

Theorem 15 was proved in $[4,22,31]$. Again, the proof here is much shorter (in spite of the detailed calculations) now that we have the groundwork in Sect. 4.

Theorems 13 and 15 show that, for $c=1$, the A-optimal designs are very different from the D-optimal designs as either of $b$ or $k$ increases.

\section{Comments and Conclusions}

\subsection{Summary}

In this paper, we have concentrated on using properties of the Levi graph to find the Doptimal block designs and A-optimal block designs when the number of observations is close to minimal. We believe that the results in Sect. 4 are new. Moreover, they do not depend on the number of observations being small. They show that, if the cycle rank is at least 1 , then the Levi graph of a D-optimal design does not contain any bridge, except, possibly, one whose treatment-vertex is a leaf. Moreover, in the Levi graph of a D-optimal design with cycle rank at least 1 , no treatment-vertex is a cut-vertex. The Levi graph of an A-optimal design with cycle rank at least 1 can have no more than one treatment-vertex which is a cut-vertex, and any such vertex must be contained in a cycle.

Sections 5 and 6 use the results of Sect. 4 to give unified, and shorter, proofs of a number of known results about D-optimal and A-optimal designs with low cycle rank. Parts of [4] referred to a preliminary version of the current paper for details of the proofs.

For small designs with cycle rank 1 , the same design can be both D-optimal and A-optimal. For example, when $b=k=4$ and $v=16$, then the design in Fig. 21 is D-optimal, by Theorem 13, and A-optimal, by Theorem 15.

However, as the number of treatments increases, the A-optimal designs diverge from the D-optimal designs. For example, when $b=6, k=4$ and $v=18$, then

$$
\begin{array}{|c|c|c|c|}
\hline 1 & 2 & 3 & 4 \\
2 & 3 & 4 & 1 \\
5 & 7 & 9 & 11 \\
6 & 8 & 10 & 12 \\
\hline
\end{array}
$$

Fig. 21 A block design with $b=k=4$ and $v=12$ which is both D-optimal and A-optimal 
the design in Fig. 1a is D-optimal, by Theorem 13, but the design in Fig. 1b is A-optimal, by Theorem 15. The geometric means of the non-trivial eigenvalues of the information matrices of these two designs are 0.8411 and 0.8075 , respectively. Their harmonic means are 0.5829 and 0.6000 , respectively.

\subsection{What About Dual Designs?}

If $d$ is a block design, then its dual block design $d^{\top}$ is made by simply interchanging the roles of treatments and blocks. In the case that all treatments have the same replication, in addition to all blocks having the same size, John and Mitchell proved in [18] that $d^{\top}$ is D-optimal if and only if $d$ is $D$-optimal, and that $d^{\top}$ is A-optimal if and only if $d$ is $A$-optimal. Thus if we are interested in estimating block effects, and use equireplicate designs, then the designs which are optimal for block effects are the same as those which are optimal for treatment effects, under either the D or the A criterion.

In most experimental situations, both management constraints and randomization concerns lead us to use blocks of equal size, even though there is no such restriction on treatment replication. We are not usually interested in estimating block effects, so we are not concerned about the optimality of $d^{\top}$. Suppose that we are interested in block effects, as well as treatment effects. Since the Levi graph of $d^{\top}$ is the same as the Levi graph of $d$, Theorem 3 shows that $d^{\top}$ is D-optimal if and only if $d$ is D-optimal. Thus, whether or not we insist on equal replication for treatments, the design which is D-optimal for treatment effects is D-optimal for block effects.

The same is true for A-optimality when the cycle rank is 0 . Theorem 14 shows that the A-optimal designs are precisely the star designs. The dual of a star design has one complete block, and all other blocks of size one. Therefore a star design gives the minimal sum of resistance distances between pairs of block-vertices.

However, the same is not true for A-optimality in general. For example, consider cycle rank 1 . When $k=2$ and $b=v$, an investigation like the one in the proof of Theorem 15 shows that, for $b \geq 5, d^{\top}$ is A-optimal if and only if $d$ is in $\mathscr{C}(b, 2,2)$.

\subsection{What About Larger Cycle Rank?}

It seems unlikely that the detailed investigations in Sects. 5 and 6 can be replicated for larger cycle ranks. However, Section 6 of [1] does suggest that, when average replication is no more than three, the A-optimal designs will diverge from the Doptimal designs as $v$ increases. Thus John and Mitchell's assumption in [18] that optimal designs will always be equireplicate if possible is no longer tenable. We recommend that any computer search for optimal block designs should not rule out considering designs with unequal (possibly very unequal) replication of treatments.

Acknowledgements Part of this work was done while the authors were at the Isaac Newton Institute for Mathematical Sciences, Cambridge, UK, during the 2011 programme on Design and Analysis of Experiments.

Open Access This article is licensed under a Creative Commons Attribution 4.0 International License, which permits use, sharing, adaptation, distribution and reproduction in any medium or format, as long as you give 
appropriate credit to the original author(s) and the source, provide a link to the Creative Commons licence, and indicate if changes were made. The images or other third party material in this article are included in the article's Creative Commons licence, unless indicated otherwise in a credit line to the material. If material is not included in the article's Creative Commons licence and your intended use is not permitted by statutory regulation or exceeds the permitted use, you will need to obtain permission directly from the copyright holder. To view a copy of this licence, visit http://creativecommons.org/licenses/by/4.0/.

\section{References}

1. Bailey RA (2007) Designs for two-colour microarray experiments. Appl Stat 56:356-394

2. Bailey RA (2009) Variance and concurrence in block designs, and distance in the corresponding graphs. Mich Math J 58:105-124

3. Bailey RA, Cameron Peter J (2009) Combinatorics of optimal designs. In: Huczynska S, Mitchell JD, Roney-Dougal CM (eds) Surveys in combinatorics 2009. London Mathematical Society lecture note series, vol 365. Cambridge University Press, Cambridge, pp 19-73

4. Bailey RA, Cameron PJ (2013) Using graphs to find the best block designs. In: Beineke LW, Wilson RJ (eds) Topics in structural graph theory. Cambridge University Press, Cambridge, pp 282-317

5. Bailey RA, Schiffl K, Hilgers R-D (2013) A note on robustness of D-optimal block designs for twocolour microarray experiments. J Stat Plan Inference 143:1195-1202

6. Balabanian N, Bickart TA (1969) Electrical network theory. Wiley, Hoboken

7. Balasubramanian K, Dey A (1996) D-optimal designs with minimal and nearly minimal number of units. J Stat Plan Inference 52:255-262

8. Bapat RB, Dey A (1991) Optimal block designs with minimal number of observations. Stat Probab Lett 11:399-402

9. Bose RC (1947) Presidential address. In: Proceedings of the thirty fourth Indian Science Congress

10. Cheng C-S (1981) Graph and optimum design theories-some connections and examples. In: Bulletin of the International Statistical Institute. Proceedings of the 43rd Session (Buenos Aires), vol 49, no 1, pp 580-590

11. Cheng C-S (1981) Maximizing the total number of spanning trees in a graph: two related problems in graph theory and optimum design theory. J Comb Theory Ser B 31:240-248

12. Coxeter HSM (1950) Self-dual configurations and regular graphs. Bull Am Math Soc 56:413-455

13. Dey A, Shah KR, Das A (1995) Optimal block designs with minimal and nearly minimal number of units. Stat Sin 5:547-558

14. Gaffke N (1978) Optimale Versuchsplanung für Zwei-Faktor Modelle. Ph.D. thesis, RheinischWestfälische Technische Hochschule, Aachen

15. Gaffke N (1981) Connected graphs with a minimal number of spanning trees. J Comb Theory Ser B 30:166-183

16. Gaffke N (1982) D-optimal block designs with at most six varieties. J Stat Plan Inference 6:183-200

17. Grünbaum B (2006) Configurations of points and lines. In: Davis C, Ellers EW (eds) The coxeter legacy: reflections and projections. American Mathematical Society, Providence, pp 179-225

18. John JA, Mitchell TJ (1977) Optimal incomplete block designs. J R Stat Soc B 39:39-43

19. Jones B, Eccleston JA (1980) Exchange and interchange procedures to search for optimal designs. J R Stat Soc B 42:238-243

20. Kerr MK, Churchill GK (2001) Experimental design for gene expression microarrays. Biostatistics 2:183-201

21. Kirchhoff G (1847) Über die Auflösung der Gleichenung, auf welche man bei der Untersuchung der linearen Verteilung galvanischer Ströme gefürt wird. Ann Phys Chem 72:497-508

22. Krafft O, Schaefer M (1997) A-optimal connected block designs with nearly minimal number of observations. J Stat Plan Inference 65:375-386

23. Latif AHMM, Bretz F, Bremmer E (2009) Robustness considerations in selecting efficient two-colour microarray designs. Bioinformatics 25:2355-2361

24. Levi FW (1942) Finite geometrical systems. University of Calcutta, Kolkata

25. Mandal NK, Shah KR, Sinha BK (1991) Uncertain resources and optimal designs: problems and perspectives. Calcutta Stat Assoc Bull 40:267-282

26. Paterson L (1983) Circuits and efficiency in incomplete block designs. Biometrika 70:215-225 
27. Paterson L, Wild P (1986) Triangles and efficiency factors. Biometrika 73:289-299

28. Patterson HD, Williams ER (1976) Some theoretical results on general block designs. Congr Numer 15:489-496

29. Pükelsheim F (2006) Optimal design of experiments. Society for Industrial and Applied Mathematics, Philadelphia

30. Sachs H (1976) On the number of spanning trees. In: Sheehan J, Nash-Williams CSt-JA (eds) Proceedings of the fifth British Combinatorial Conference, University of Aberdeen, Aberdeen, July 14-18, 1975, pp 529-535

31. Sajjad A (2011) Optimality in sparse block designs. Ph.D. thesis, Quaid-i-Azam University, Islamabad

32. Shah KR, Sinha BK (1989) Theory of optimal designs. Lecture notes in statistics, vol 54. Springer, New York

33. Tjur T (1991) Block designs and electrical networks. Ann Stat 19:1010-1027

34. Tjur T (1993) An algorithm for optimization of block designs. J Stat Plan Inference 36:277-282

35. Wild P (1987) On circuits and optimality conjectures for block designs. J R Stat Soc B 49:90-94

36. Wit E, Nobile A, Khanin R (2005) Near-optimal designs for dual-channel microarrays. Appl Stat 54:817-830

Publisher's Note Springer Nature remains neutral with regard to jurisdictional claims in published maps and institutional affiliations. 